\section{Comment on "Direction of Optical Energy Flow in a Transverse Magnetic Field"}

In a recent article [1] Rikken and van Tiggelen posed the question whether the direction of the energy transport of light is always given by the direction of the Poynting vector, $\mathbf{S}=\mathbf{E} \times \mathbf{H}$. They performed two experiments; the first one was to show the sensitivity of the measuring experimental equipment, the second to give evidence for the fact that the direction of the energy flow in an absorbing, circular dichroic material was not directed along the calculated direction of $\mathbf{S}$, knowing the various elements of the dielectric tensor. Our Comment concerns mainly the first experiment. There the deflection of a light beam was measured while traversing a nonabsorbing material, with a nonzero Verdet constant, subject to an external magnetic field perpendicular to the direction of the incoming light beam. The deflection in the direction of the magnetic field was monitored. The authors state for this geometry that circularly polarized waves are the eigenmodes of the system, and therefore, measured the difference in deflection between two circularly components. We assert that for a Hermitian dielectric tensor and exactly perpendicular alignment of magnetic field with respect to the wave vector (the Voigt symmetry) the two eigenmodes are both linearly polarized, one parallel and one perpendicular to the magnetic field, and furthermore, that the light will undergo no deflection. This latter point already follows from symmetry considerations: the wave vector and its deflection are polar vectors, while the magnetic field is an axial vector. Applying inversion symmetry with respect to a plane parallel to the wave vector but perpendicular to the magnetic field will change sign while the magnetic field itself and the linear polarizations of the eigenmodes remain unchanged. This deflection must therefore be zero. This is consistent with the fact that the Cotton-Mouton effect is quadratic in the magnetic field strength.

Solving the Helmholtz equation for a system with angle $\phi$ between magnetic field $\mathbf{B}$ and the wave vector, and calculating the angle $\theta$ between the Poynting vectors of the two eigenmodes, one arrives at

$$
\theta=2 \arctan \frac{b \sin \phi \cos \phi}{\sqrt{b^{2} \sin ^{4} \phi+4 \cos ^{2} \phi}},
$$

with $b=\gamma B / \varepsilon$, and where $\gamma$ is the parameter describing the strength of the Faraday effect, and $\varepsilon$ the isotropic, field-free dielectric constant. When the external magnetic field lies, e.g., in the $y z$ plane and the wave vector points along the $z$ axis, the exact expression for the electric displacement $\mathbf{D}=\boldsymbol{\varepsilon} \cdot \mathbf{E}$ of an eigenmode reads $\mathbf{D} \propto$ $\left(-2 i \cos \phi, b \sin ^{2} \phi+\sqrt{b^{2} \sin ^{4} \phi+4 \cos ^{2} \phi}, 0\right)$. For the other mode the $x$ and $y$ components are interchanged.
It should be noted that the Poynting vector describes the energy flow correctly for nonabsorbing media with a Hermitian dielectric tensor (see, e.g., Ref. [2]). In this case the Poynting vector gives the same result as the group velocity. From Eq. (1) it is seen that for angles $\phi$ not too close to $\pi / 2$, i.e., $\cos \phi \gg b \sin ^{2} \phi$, the resulting deflection angle simply becomes

$$
\theta \approx \frac{\gamma B}{\varepsilon} \sin \phi .
$$

In this approximation the eigenmodes resemble closely circularly polarized waves, the deviations being only of the order of $(\gamma B / \varepsilon) \sin \phi$.

Exactly in the Voigt symmetry, however, not the circularly polarized but the linearly polarized waves are the true eigenmodes of the system, in contrast to the arguments of Rikken and van Tiggelen, who considered only the first order in $\gamma B / \varepsilon$. For one mode the electric displacement $\mathbf{D}$ is parallel to the external magnetic field. For the second mode $\mathbf{D}$ is perpendicular to both the external magnetic field as well as the wave vector. For this latter mode the electric field has a small component (of the order of $\gamma B / \varepsilon$ ) along the direction of the wave vector. Furthermore, $\cos \phi=0<\gamma B / \varepsilon$. Consequently, the deflection angle $\theta$ is zero. The measurements of Rikken and van Tiggelen could be explained, if the magnetic field and the wave vector were not exactly perpendicular, but instead had a misalignment of at least $\gamma B / \varepsilon$. For their experimental conditions this misalignment is rather small, about $10^{-7} \mathrm{rad}$. This exemplifies the difficulty in obtaining correctly interpretable experimental observations concerning optical energy flow in anisotropic media. Energy transport in absorbing media, where no relation between Poynting vector and group velocity has been derived, poses even more difficulties.

J.C. J.P. acknowledges support by the Dutch Science Foundation NWO.

\author{
G. W. 't Hooft, ${ }^{1,2}$ G. Nienhuis, ${ }^{2}$ and J.C. J. Paasschens ${ }^{1,3}$ \\ ${ }^{1}$ Philips Research Laboratories \\ 5656 AA Eindhoven, The Netherlands \\ ${ }^{2}$ Huygens Laboratory, University of Leiden \\ 2300 RA Leiden, The Netherlands \\ ${ }^{3}$ Instituut-Lorentz, University of Leiden \\ 2300 RA Leiden, The Netherlands
}

Received 22 September 1997

[S0031-9007(97)05150-8]

PACS numbers: 42.25.Bs, 42.81.Gs, 78.20.Ls

[1] G. L. J. A. Rikken and B. A. van Tiggelen, Phys. Rev. Lett. 78, 847 (1997).

[2] H. Haus, Waves and Fields in Optoelectronics (PrenticeHall, Englewood Cliffs, NJ, 1984). 